\title{
A NOVEL GENERALIZED IMPEDANCE CONVERTER USING SINGLE SECOND GENERATION CURRENT CONVEYOR
}

\author{
IQBAL A. KHAN ${ }^{\mathrm{a} *}$ and MEHMOOD H. ZAIDI ${ }^{\mathrm{b}}$ \\ ${ }^{a}$ Department of Electronics Engineering, Aligarh Muslim University, Aligarh-202002, India; \\ ${ }^{\mathrm{b}}$ Department of Electrical Engineering, Faculty of Engineering and Technology, \\ Jamia Millia Islamia, Jamia Nagar, Okhla, New Delhi-110025
}

(Received 18 August 2002; In final form 17 November 2002)

\begin{abstract}
A generalized impedance converter (GIC) using single CCII(-) is presented. The circuit uses no matching constraints and provides a wide variety of ideal component simulators and multipliers. The GIC can also be made tunable by using CCCII $(-)$ instead of CCII $(-)$. The proposed GIC is verified using SPICE with excellent results.
\end{abstract}

Keywords: Active filters; Current conveyors; Impedance converters

\section{INTRODUCTION}

In recent years the second-generation current conveyor (CCII) has proved to be a functionally flexible and versatile building block. It possesses higher signal bandwidth, greater linearity and dynamic range [1]. As a result, it is gaining wide acceptance as a basic building block for designing voltage/current mode signal processing circuits [1-7].

Several circuits realizing immittance functions using CCIIs have been reported [8-11]. However, these circuits either provide non-ideal simulators and/or use component matching constraints.

In this paper a generalized impedance converter (GIC) using a $\mathrm{CCII}(-)$ is presented. The proposed GIC is in grounded form and provides an ideal impedance simulator without any matching constraints. The realized GIC is studied and verified using SPICE based simulations.

\section{CIRCUIT REALIZATION}

The proposed GIC using a CCII(-) is given in Figure 1. The routine analysis yields its driving point impedance function as:

$$
Z_{\text {in }}=\frac{V}{I}=\frac{Z_{1} Z_{3}}{Z_{2}}
$$

* Corresponding author. E-mail: iqbal.a.khan@mailcity.com 


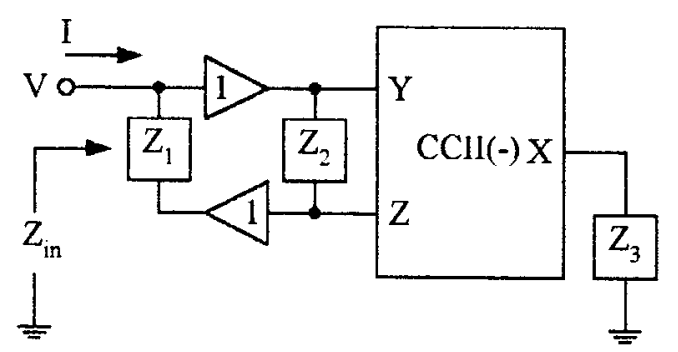

FIGURE 1 A GIC using single CCII(-).

By appropriate selection of $Z_{1}, Z_{2}$ and $Z_{3}$ in (1), the GIC can realize ideal grounded inductance, capacitance/resistance multipliers and frequency-dependent negative resistance (FDNR). The proposed GIC possesses the incremental sensitivity measure as:

$$
\left|S_{Z_{j}}^{Z_{\text {in }}}\right|=1.0, \text { where } j=1,2 \text { and } 3 .
$$

It is evident from (1) that the GIC also enjoys the attractive sensitivity performance. In Figure 1 if the CCII $(-)$ along with $Z_{3}$ is replaced by a second generation current controlled conveyor CCCII(-) as shown in Figure 2, the resulting input impedance is

$$
Z_{\text {in }}=\frac{V}{I}=\frac{Z_{1} R_{x}}{Z_{2}}
$$

where $R_{x}\left(=V_{T} / 2 I_{0}\right)$ is the parasitic resistance at the $x$-input of the CCCII(-). The $V_{T}$ is thermal equivalent voltage and $I_{0}$ is the bias current $[12,13]$.

It is evident from (1) that the GIC of Figure 2 can realize all the immittance functions simulated from the GIC of Figure 1 except FDNR. It is to note that the CCCII(-) based GIC of Figure 2 uses only two passive components and possesses a wide range tuning facility through bias current control. It also enjoys similar sensitivity performance to that of CCII $(-)$ based GIC of Figure 1.

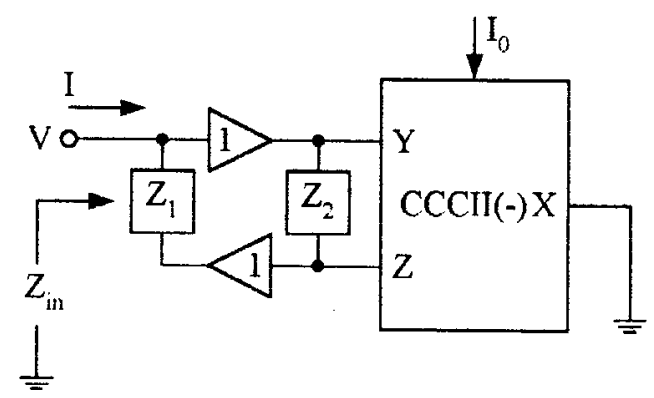

FIGURE 2 A GIC using single CCCII(-). 


\section{EFFECTS OF NON-IDEAL VOLTAGE BUFFER}

If the voltage buffers have slight gain deviations $\delta_{1}$ and $\delta_{2}$ from unity, then the input impedance function of Figure 1 is:

$$
Z_{\text {in }}=\frac{Z_{1}}{\left\{1-\left(1-\delta_{1}\right)\left(1-\delta_{2}\right)\left(1-Z_{2} / Z_{3}\right)\right\}}
$$

and that of Figure 2 is:

$$
Z_{\text {in }}=\frac{Z_{1}}{\left\{1-\left(1-\delta_{1}\right)\left(1-\delta_{2}\right)\left(1-Z_{2} / R_{x}\right)\right\}} .
$$

It is evident from (4) and (5) that because of this non-ideality the actual values of the simulated components are slightly affected.

The incremental sensitivity measure of input impedance functions as given by (4) and (5) with respect to the gain deviation $\delta$ (with $\delta_{1}=\delta_{2}=\delta$ ) of the voltage buffer when used in (1) and (2) as:

$$
S_{\delta}^{Z_{\text {in }}}=\frac{2 \delta(1-\delta)}{\left\{1-(1-\delta)^{2}\left(1-Z_{2} / Z_{3}\right)\right\}}\left(1-\frac{Z_{2}}{Z_{3}}\right) .
$$

It is evident from (6) that the incremental sensitivity of (4) and (5) with respect to gain deviation $\delta$ of the voltage buffers is found to be low in magnitude.

\section{SPICE SIMULATION AND VERIFICATIONS}

The working ability of the GIC is presented here and has been confirmed by SPICE simulations. The SPICE model of the current conveyor of reference [3] was used in the simulation. The voltage and the current wave shapes across an ideal inductor of $0.1 \mathrm{H}$ realized from GIC of Figure 1 with $Z_{1}=Z_{2}=R=10 \mathrm{k} \Omega$ and $Z_{2}=1 / s C_{2}$ with $C_{2}=1.0 \mathrm{nF}$ are shown in Figure 3, which shows a good agreement with the theory.

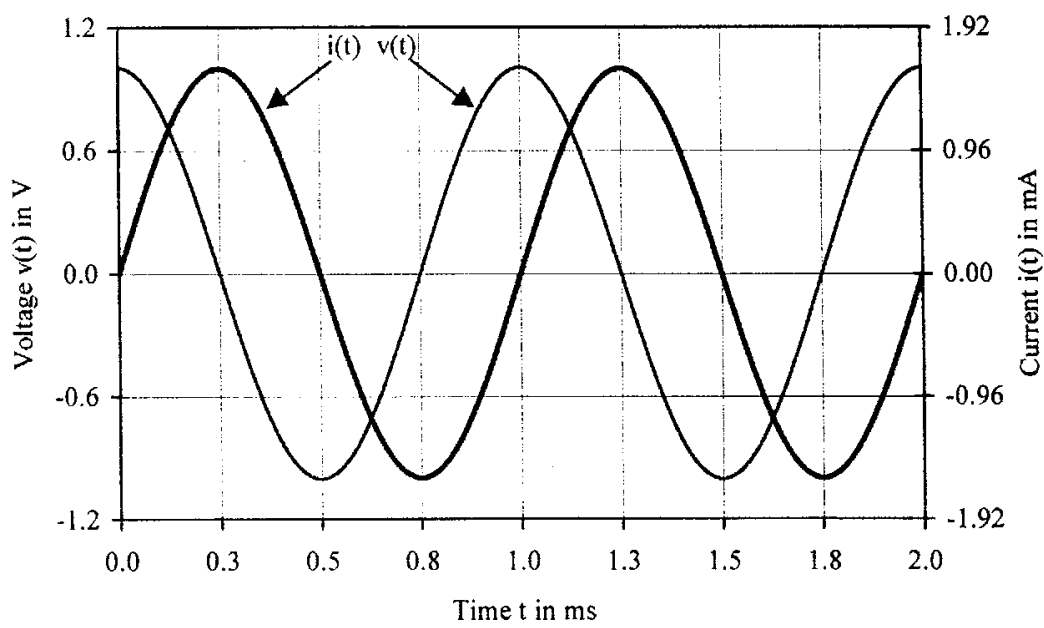

FIGURE 3 Voltage $v(t)$ and current $i(t)$ wave shapes across the simulated ideal grounded inductor from GIC of Figure 1. 


\section{CONCLUSIONS}

A new generalized impedance converter using single CCII(-) is presented. This GIC is capable of realizing ideal grounded inductor, capacitor/resistor multipliers and frequencydependent negative resistance. The GIC presented also enjoys the advantages of realization of ideal simulators without any matching constraints. The GIC can also be made tunable over a wide range for simulated parameters by replacing CCII(-) with $\mathrm{CCCII}(-)$. The SPICE simulated results of GIC verify the theory. However, by adding the tuning facility the GIC loses the capability for FDNR realization. The effects of non-ideal voltage buffers on the realized impedance functions are also examined.

\section{References}

[1] Wilson, B. (1990). Recent developments in current conveyors and current mode circuits. IEE Proceedings-G, 132(2), 63-73.

[2] Wilson, B. (1992). Tutorial review: Trends in current conveyors and current mode amplifier design. Int. J. Electronics, 73(3), 573-583.

[3] Brun, E. and Olesen, O. H. (1992). Conveyors implementations of generic current mode circuits. Int. J. Electronics, 73(1), 129-140.

[4] Hou, C. L., Chen, R. D., Wu, Y. P. and Hu, P. C. (1993). Realizations of grounded and floating immittance function simulators using current conveyors. Int. J. Electronics, 74(6), 917-923.

[5] Sun, Y. and Fidler, J. F. (1994). Versatile active biquad based on second generation current conveyors. Int. J. Electronics, 76(1), 91-98.

[6] Abuelma'atti, M. T. and Khan, M. H. (1995). Low component current mode universal filter. Electronics Letters, 31(25), 2160-2161.

[7] Soliman, A. M. (1997). Theorems relating to port interchanging in current mode CCII circuits. Int. J. Electronics, 82(6), 585-604.

[8] Soliman, A. M. (1978). New active gyrator using a single current conveyor. Electronics Letters, 66, 1580-1581.

[9] Soliman, A. M. (1978). Realization of frequency dependent negative resistance circuits using two capacitors and a single current conveyor. IEE Proceedings- $G, \mathbf{1 2 5}, 1336-1337$.

[10] Nedungadi, A. (1979). Comment: Active simulation of inductors using current conveyor. Electronics Letters, 15(4), 112-113.

[11] Paul, A. N. and Patranabis, D. (1981). Active simulation of grounded inductor using a single current conveyor. IEEE. Transactions on Circuits and Systems, CAS-28, 164-165.

[12] Fabre, A., Saaid, O., Wiest, F. and Boucheron, C. (1995). Current controlled band pass filter based on translinear conveyors. Electronics Letters, 31(20), 1727-1728.

[13] Khan, I. A. and Zaidi, M. H. (2000). Multifunctional translinear-C current mode filter. Int. J. Electronics, 87(9), $1047-1051$. 

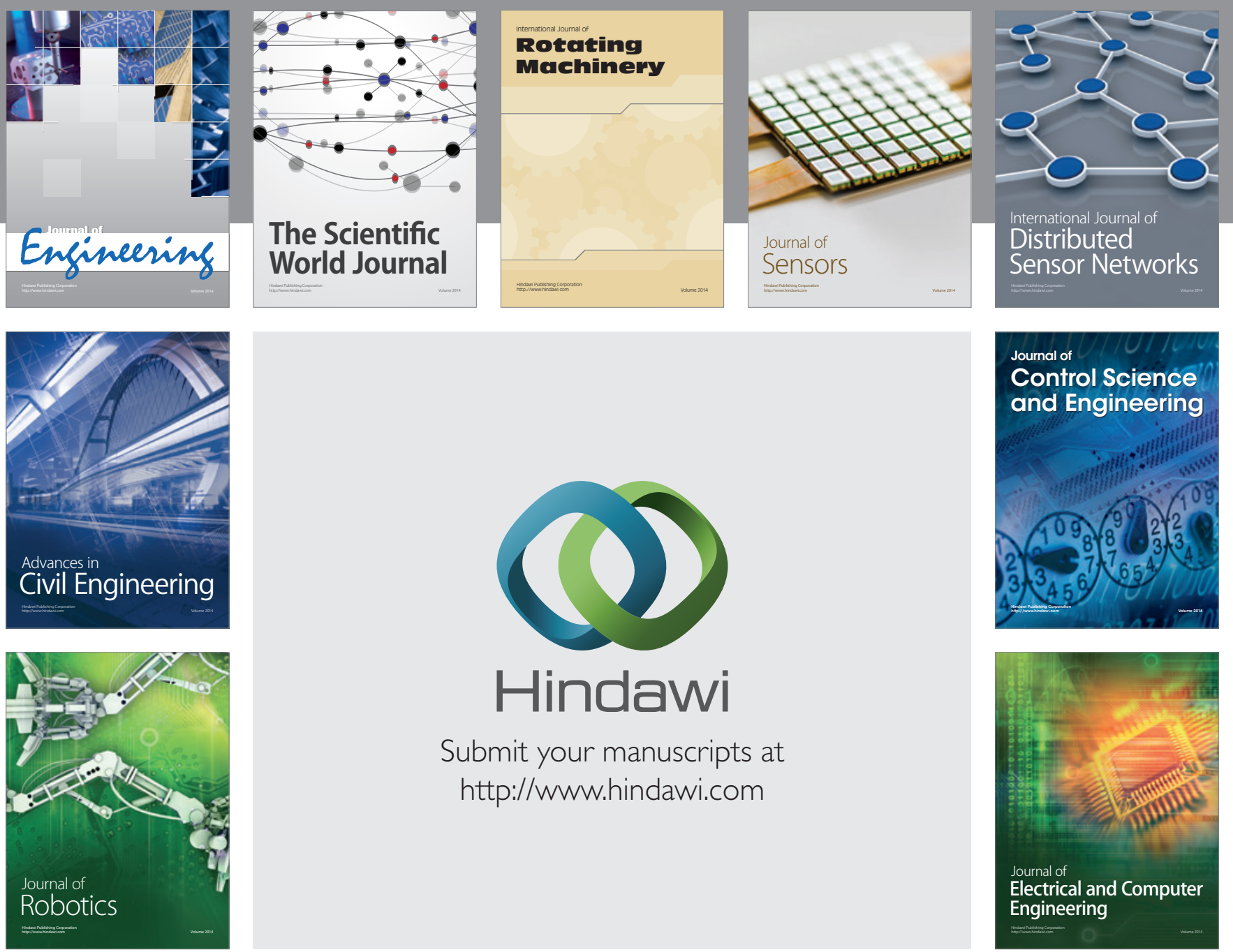

Submit your manuscripts at

http://www.hindawi.com
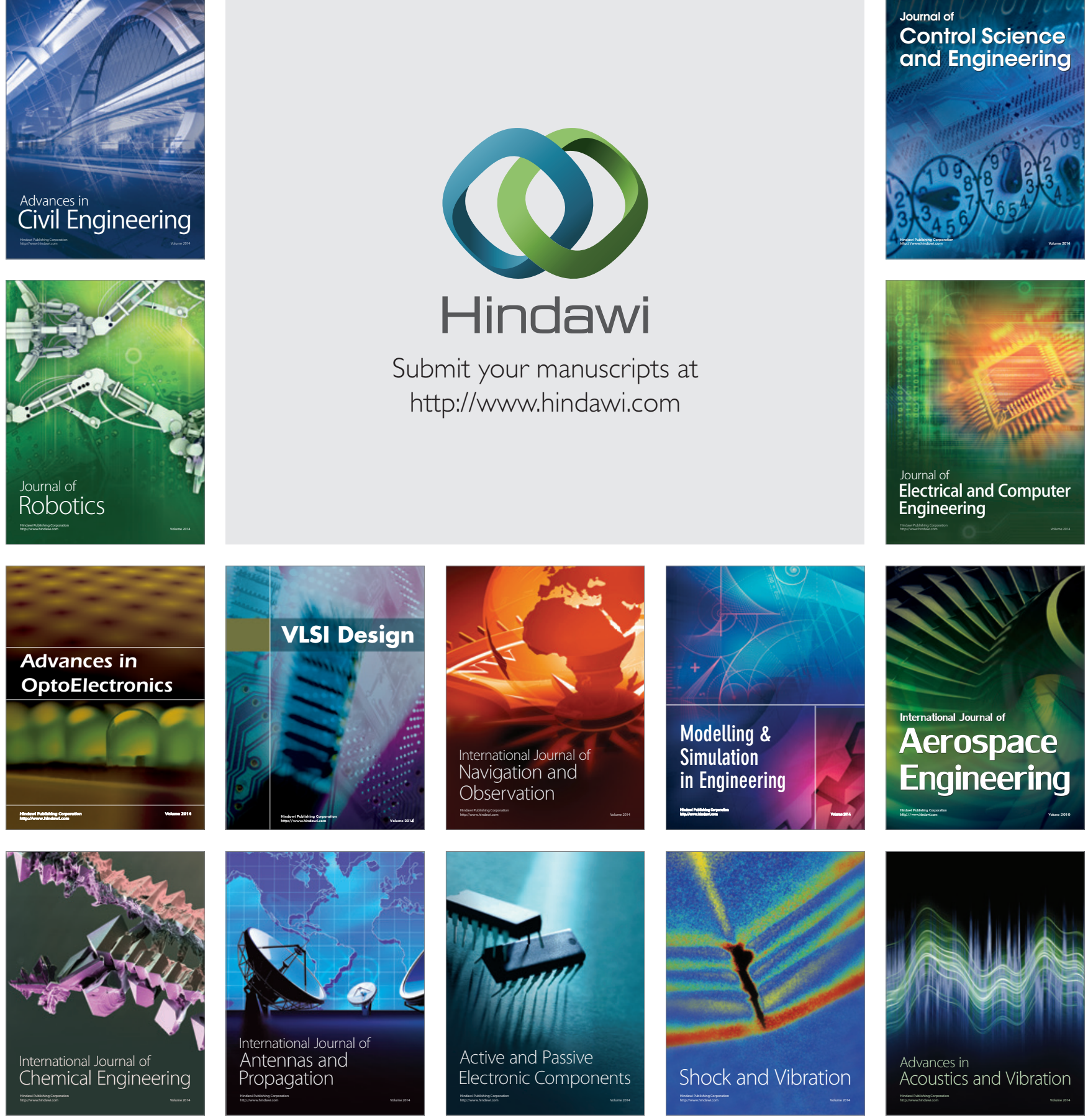